\title{
Mifegest and Misoprostol Combination Vs Misoprostol Alone for Termination of Second Trimester of Pregnancy
}

\author{
Author \\ Rashmi Verma \\ Dept of Obsterics and Gynaecology, Katihar Medical College and Hospital, Katihar, Bihar, India \\ Corresponding Author \\ Rashmi Verma \\ Flat No. - 401, Sukh-Smriti Apartment, Near Mangal market, Sheikhpura, \\ Bailey Road, Patna-800014, Bihar, India \\ Email:ais_25@yahoo.com
}

\begin{abstract}
Objective: Over the last few years, induced abortions have gained more popularity because of safe techniques and medications available. Induced abortion means will ful termination of pregnancy before the period of viability. Medical abortion in the second trimester with misoprostol alone has been shown to be affective, although in comparison with the combination of mifepristone and misoprostol, misoprostol-only protocols have required higher doses, side effects are more common and the time to complete the abortion is longer.

Methods: Total of 50 eligible women were enrolled for this study and were divided in two groups of 25 each of the case group and control group. This study was conducted in Obstetrics and gynaecology department of Katihar Medical College and Hospital, Bihar. Women in the case group were given Tablet

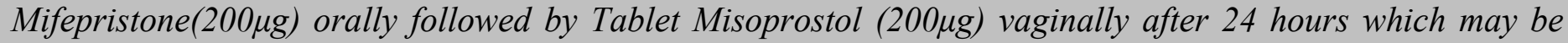

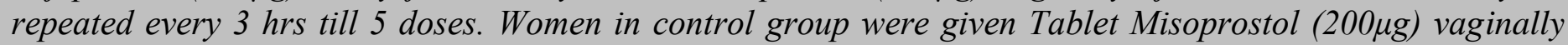
which may be repeated every 3 hrs till 5 doses.

Results: The combination of mifepristone with misoprostol significantly reduces the abortion to induction interval and also have fewer side effects and complications and also reduces the dose of misoprostol. Where mifepristone is not available or affordable, misoprostol alone has also been shown to be effective, although a higher total dose is needed and efficacy is lower than for the combined regimen. Therefore, whenever possible, the combined regimen should be used.

Conclusion: Mifepristone followed by misoprostol was more effective and has a shorter Induction abortion interval and fewer side effects.

Keywords: Induced Abortion, Second trimester, Mifepristone, Misoprostol
\end{abstract}

\section{Introduction}

There is a need for evolving a safe and effective method of terminating pregnancy in the second trimester, most recently due to the increase in the use of antenatal diagnostic procedures like amniocentesis, USG and cordocentesis. Induced abortion means willful termination of pregnancy before the period of viability. Second trimester abortions constitute $10-15 \%$ of all induced abortions worldwide but are responsible for twothirds of major abortion-related complications. In India, 6 million abortions takes place every year, 
of which 4 million are induced and 2 million are spontaneous. Although abortions was legalized in India is 1972, illegal abortion is still five times more common than legal abortion. Medical abortion in the second trimester with misoprostol alone has been shown to be affective, although in comparison with the combination of mifepristone and misoprostol, misoprostol-only protocols have required higher doses, side effects are more common and the time to complete the abortion is longer.5Higher doses have shown comparable successful abortion rates but are associated with higher rates of side effects. The 3-hour interval is more effective than 6 hours interval.Regimen of mifepristone and misoprostol has been found safe and effective in both developed and developing countries for 2nd trimester termination of pregnancy.

\section{Materials and Methods}

1. To evaluate the efficacy of tablet Mifepristone in combination with tablet Misoprostol in management of second trimester abortion and compare it with Misoprostol alone.

2. To observe the course and outcome of abortion in this combined regimen.

3. To study the possible side effects of these drug Total of 50 eligible women were enrolled for this study and were divided in two groups of 25 each. Case: Women who received Mifepristone and Misoprostol.

Control: Women who received Misoprostol alone.

\section{Inclusion Criteria:}

1. Gestational age more than 16 weeks but less than 20 weeks.

2. Singleton pregnancy

3. No regular uterine contractions

4. Upto para 4.

\section{Exclusion Criteria:}

1. Grand multipara

2. Scarred uterus

3. Multiple pregnancy

4. Heart disease or known contraindication to the use of study drugs
Study Design: All the eligible patients were explained about the procedure and their written informed consent was taken. Women in the case group were given Tablet Mifepristone $(200 \mu \mathrm{g})$ orally followed by Tablet Misoprostol $(400 \mu \mathrm{g})$ vaginally after 24 hours which may be repeated every 3 hrs till 5 doses. Women in control group were given Tablet Misoprostol $(400 \mu \mathrm{g})$ vaginally which may be repeated every 3 hrs till 5 doses.
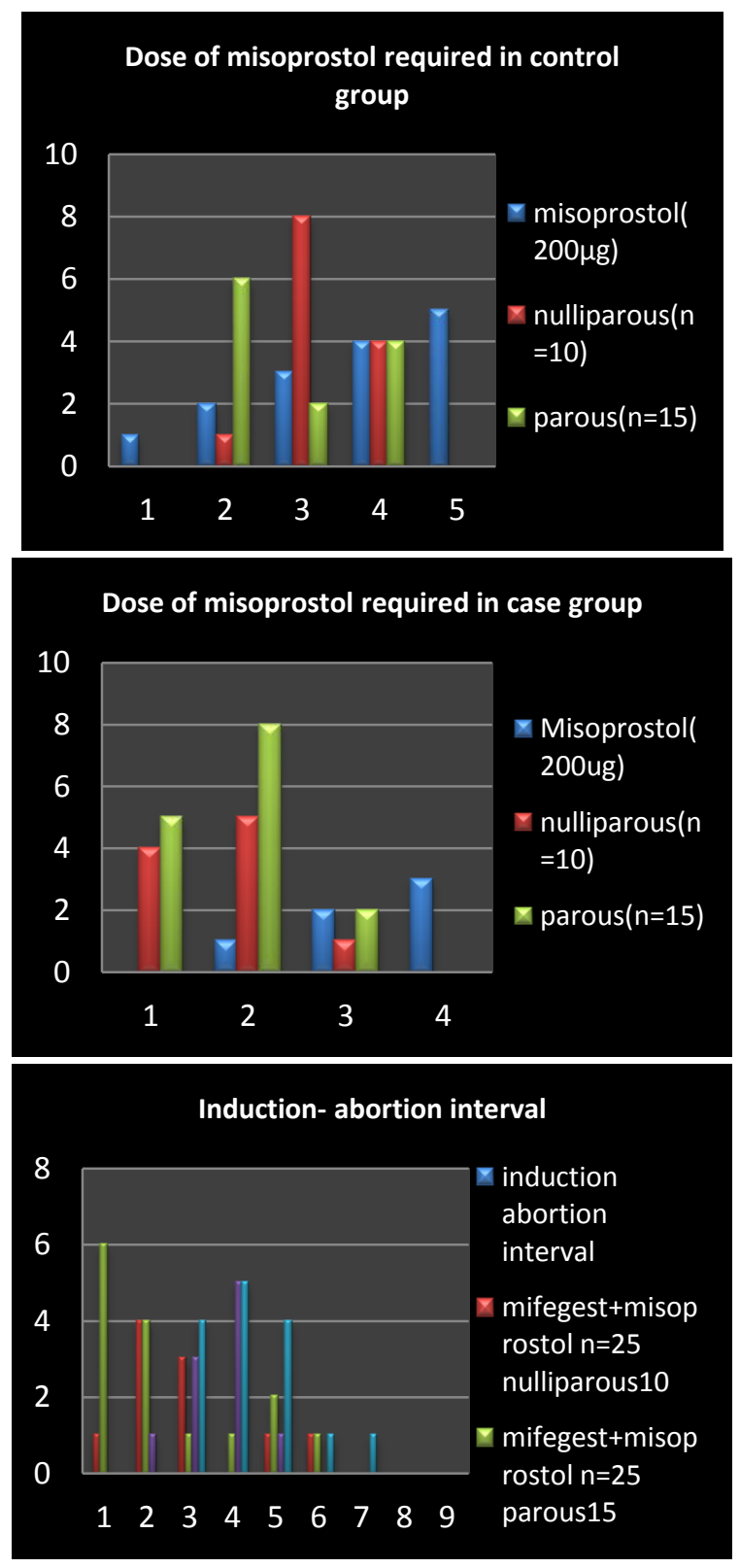

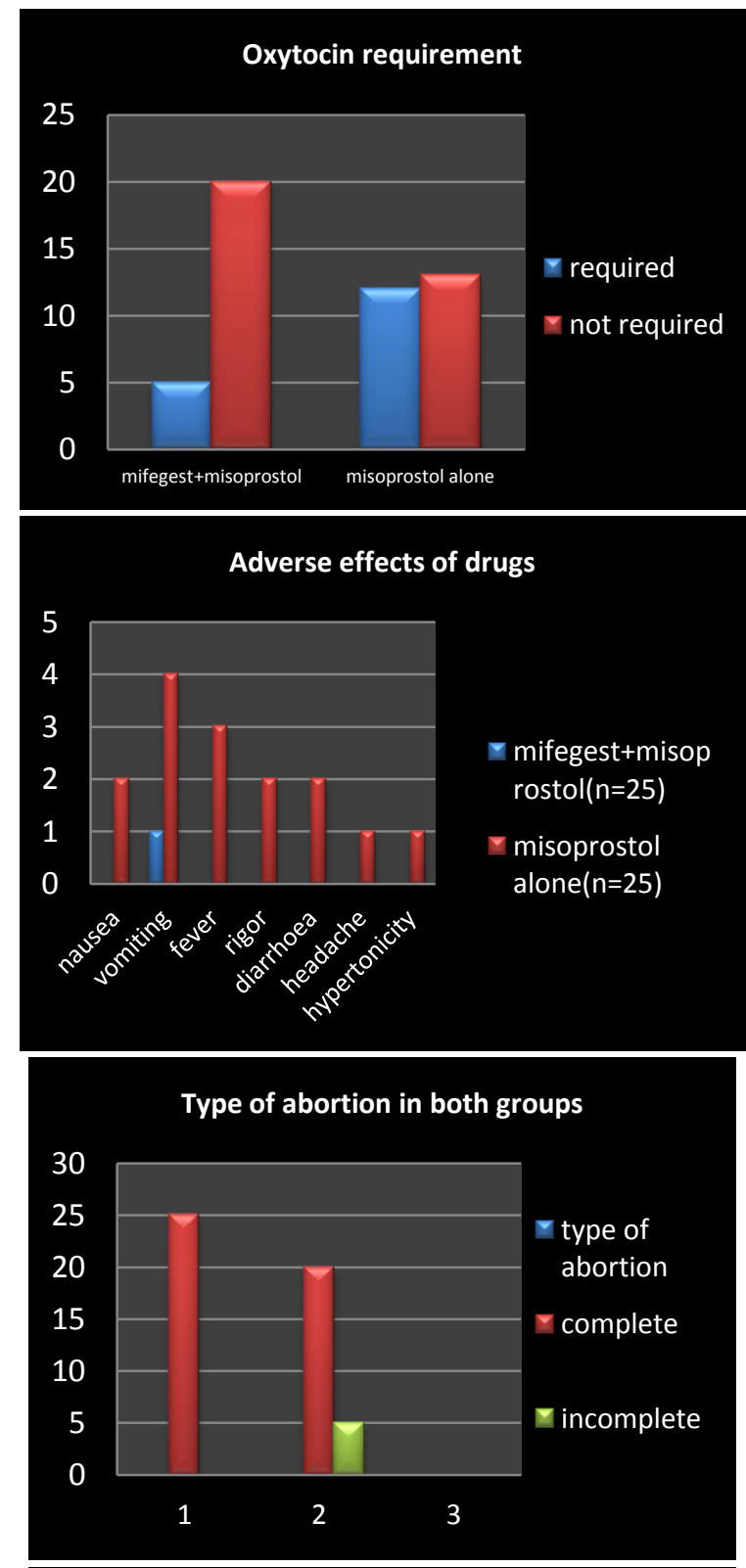

Duration of hospital stay in both groups

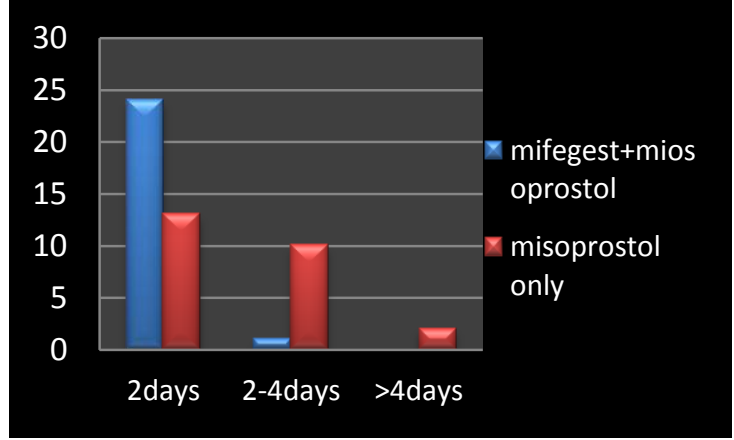

\section{Results}

There were total 10 Nulliparous and 15 parous women taken in the Control group for the study. There was no woman in either nulliparous or parous group who required just 1 misoprostol tablet $(1 \mathrm{misoprostol}$ tablet $=200 \mu \mathrm{g})$ for abortion. 1 women from nulliparous group and 6 women from parous group required 2 misoprostol tablets for abortion. There were 8 nulliparous and 2 women from parous group who required 3 misoprostol tablets for abortion. 4 women from nulliparous group and 4 women from parous group who required 4 misoprostol tablets for abortion. There was no woman in either group who required 5 misoprostol tablets for abortion. It was observed that complete abortion occurred in 25 patients receiving mifepristone and misoprostol both and in 20 patients receiving misoprostol alone. There was no patient who had incomplete abortion after receiving mifepristone and misoprostol and 5 patients receiving misoprostol alone.same number of nulliparous and parous women were taken in case and control group that was 10 in nulliparous and 15 in parous.In case group there were 4 nulliparous and 5 parous women who required 0 misoprostol tablet for abortion. 5 nulliparous and 8 parous required 1 tab of misoprostol and 1 nulliparous and 2 parous required 2 tab of misoprostol for abortion. Not even a single woman of either group required 3 tablets of misoprostol for abortion. There was a significant difference in the induction -abortion interval of the two groups. Requirement of oxytocin was much less in the mifegest misoprostol combination than misoprostol alone. The incidence of adverse effects was more in misoprostol alone than the combination drug. A lesser duration of hospital stay was observed in the mifegest and misoprostol group than misoprostol alone. It was observed that complete abortion occurred in all patients receiving mifegest and misoprostol than those who received misoprost alone where 20 complete and 5 incomplete abortions were seen.

\section{Discussion}

Second trimester pregnancy termination is still a complicated procedure in developing countries especially in rural areas. There is constant search going on for an ideal method which is $100 \%$ reliable, safe and cheap. The IAI, success rate, hospital stay duration, side effects, and costs are compared between the two groups. There was 
significant difference in the IAI in both the groups, being much lesser in the mifegest and misoprostop group than misoprost alone. There was also difference in the success rate and hospital stay duration. The hospital stay was longer in Group B than Group A. Side effects were also seen more in Group B than Group A. The combination of oral mifepristone $200 \mu \mathrm{g}$ pretreatment, followed by vaginally misoprostol, provides a non-invasive effective regimen for medical second trimester termination of pregnancy and significantly reduces the induction to abortion interval and lesser side effects and good patient compliance. The side effects observed were actually directly associated with the dosage of misoprostol. This method can be used in an outpatient clinic or primary health centre where facilities for surgical evacuation are not available. The doctors with back up facility who are not trained in MTP's can perform this procedure. In case of any problem like retained products, suspected abortion failure, heavy bleeding per vaginum the patient can be referred to health facilities for surgical evacuation are available. With this procedure morbidity and mortality due to illegal abortions can be markedly reduced.

\section{Conclusion}

During the last decade, medical methods for second trimester induced abortion have been considerably improved and become safe and more accessible. Today, in most cases, safe and efficient medical abortion services can be offered or improved by minor changes in existing health care facilities. Because of the potential for heavy vaginal bleeding and serious complications, it is advisable that second trimester terminations take place in a health care facility where blood transfusion and emergency surgery (including laparotomy) are available. The combination of mifepristone and misoprostol is now an established and highly effective and safe method for medical method second trimester abortion. The combination of mifepristone with misoprostol significantly reduces the abortion to induction interval and also have fewer side effects and complications and also reduces the dose of misoprostol. Where mifepristone is not available or affordable, misoprostol alone has also been shown to be effective, although a higher total dose is needed and efficacy is lower than for the combined regimen. Therefore, whenever possible, the combined regimen should be used. Efforts should be made to reduce unnecessary surgical evacuation of the uterus after expulsion of the fetus. So among the two methods, mifepristone followed by misoprostol was more effective and has a shorter IAI and fewer side effects. But in fact, both are feasible as far as end results are concerned.

\section{References}

1. The last abortion Clinic Frontline 2005. Available at www.pbs.org/wgbh/psges/frontline/clinic.

2. Chaudhary SK. Pregnancy termination. In: Chaudhary SK, editor. Practice of fertility control. 5th edition. London: Churchill Livingstone Pvt. Ltd, 2001;268-69.

3. Chhabra R, Nuna SC. Abortion in India an overview. Delhi: Ford foundation; 1994.

4. Karkal M. Abortion laws and the abortion situation in India. Reprod Genet Eng 1991;4:223-30.

5. Caliskan E, Dilbaz S, Doger E, Ozeren S, Dilbaz B. Randomized comparison of 3 misoprostol protocols for abortion induction at 13-20 weeks of gestation. J Reprod Med 2005;50(3):173-80.

6. Tang OS, Gemzell-Danielsson K, Ho PC. Misoprostol: Pharmacokinetic profiles, effects on the uterus and side-effects. Int $\mathbf{J}$ Gynaecol Obstet 2007;99:160-7.

7. Jonathan S Berecl. "Novak's Gynecology" Thirteenth edition: Lippincott Williams \& Wilkins publication, 2002:279-81. 